\title{
A Space-Time Block Coded Multiuser MIMO Downlink Transmission Scheme
}

\author{
Yi Hong \\ Inst. for Telecom. Research \\ University of South Australia \\ Email: Yi.Hong@unisa.edu.au
}

\author{
Emanuele Viterbo \\ Politecnico di Torino \\ Torino, Italy \\ Email: viterbo@polito.it
}

\author{
Jean-Claude Belfiore \\ ENST \\ Paris, France \\ Email: belfiore@enst.fr
}

\begin{abstract}
In this paper, we consider the downlink of a space time block coded multiuser multiple-input multiple-output (MIMO) system. We propose a transmission scheme to support highest possible data rate and full diversity for multiuser MIMO systems. For this, threaded algebraic space-time block codes and perfect space-time block codes are employed. Different spreading matrices are used to separate the data streams of multiple users. After despreading the signal sequence at the receiver of each user, the maximum likelihood decoding is obtained by a lattice decoder. Performance of the multiuser MIMO system in the presence of multiple access interference is evaluated by simulations in terms of block error rate.
\end{abstract}

Keywords: Perfect STBC, TAST codes, MIMO, multiuser, downlink

\section{INTRODUCTION}

Space-time coding was proposed in [1] by combining channel coding and transmit diversity techniques in order to achieve diversity and coding gains. In the coherent scenario, where the channel state information (CSI) is available at the receiver, the design criteria for full diversity space-time codes in quasistatic fading channels were developed [1]. Space-time block codes (STBCs) attract a lot interests due to the low complexity of linear decoding, full diversity gain, and high transmission rates with low delay.

Orthogonal STBCs in $[2,3]$ and diagonal algebraic spacetime (DAST) block codes in $[4,5]$ were proposed to achieve full diversity with a low complexity receiver. However, these codes do not achieve all the potential full transmission rates.

In [6], threaded algebraic space-time (TAST) block codes were proposed for arbitrary numbers of transmit and receive antennas. In [7], full rate and full rank linear dispersion STBCs were designed using cyclic division algebra.

However, the minimum determinants of the codeword difference matrix for the above constructions vanish for increasing size of the signal constellation. In [8-10], full rate and full diversity codes, named Perfect STBCs, with non-vanishing minimum determinants were proposed.

In the downlink of a multiple-input multiple-output (MIMO) multiuser system, a base station simultaneously transmits signals for many different users. Multiple antennas at both transmitters and receivers ensure high data rates, which are required in the next generation wireless communication systems. Hence it is natural to combine STBCs with downlink transmission schemes for a MIMO multiuser system. Recent work in [14] uses orthogonal STBCs in the downlink of a Direct Sequence Code Division Multiple Access (DS-CDMA) system, where the multiple-input single-output (MISO) case was considered.

In our paper, we consider CDMA as a multiplexing scheme. We show how to use TAST codes and perfect STBCs in the downlink of a MIMO multiuser system. Different orthogonal spreading matrices are used to separate the data streams of multiple users. At the receiver of each user, after despreading the received signal sequence, the maximum likelihood decoding is obtained by a lattice decoder. The performance of the downlink system is evaluated by simulation in terms of block error rate (BLER). Due to multipath propagation, different time delayed versions of the transmitted signal may result in multiple access interference (MAI). The impact of MAI on the system performance is also analyzed by simulation.

The rest of the paper is organized as follows. Section II introduces the system model. Section III presents how to apply TAST block codes and perfect STBCs to the downlink of MIMO multiuser systems. In Section IV, lattice decoding for this space-time block coded MIMO multiuser system is shown. Section V shows simulation results. Finally, conclusions are drawn in Section VI.

\section{SySTEM MODEL}

The following notations are used in the paper: $T$ denotes transpose and $\dagger$ denotes transpose conjugate. Let $\mathbb{Z}, \mathbb{Q}, \mathbb{C}$ and $\mathbb{Z}[j]$ denote the ring of rational integers, the field of rational numbers, the field of complex numbers, and the ring of Gaussian integers, where $j^{2}=-1$. Let $\mathcal{S}$ and $\mathcal{S}^{2}$ denote PAM and QAM constellation sets, respectively. Let $\mathbb{Q}(\theta)$ denote an algebraic number field generated by the primitive element $\theta$. The $m \times m$ dimensional identity matrix is denoted by $\mathbf{I}_{m}$. The matrix $\mathbf{1}_{m}$ is defined as an all ones $m \times m$ matrix. Given a $m$ dimension vector $\mathbf{v}, \mathbf{V}=\operatorname{diag}(\mathbf{v})$ is the $m \times m$ diagonal matrix with $\mathbf{V}_{i, i}=v_{i}$ and $\mathbf{V}_{i, k}=0$ for all $i, k=1, \ldots, m$, and $i \neq k$.

We consider the downlink of a $K$ user MIMO multiuser system, as shown in Fig. 1. We assume that the base station has $n_{T}$ transmit antennas, where $n_{T}=\sum_{k=1}^{K} n_{R}{ }^{(k)}$ and $n_{R}{ }^{(k)}$ denotes the number of receive antennas for $k$-th user. This enables to support terminals with different receiver complexity. 


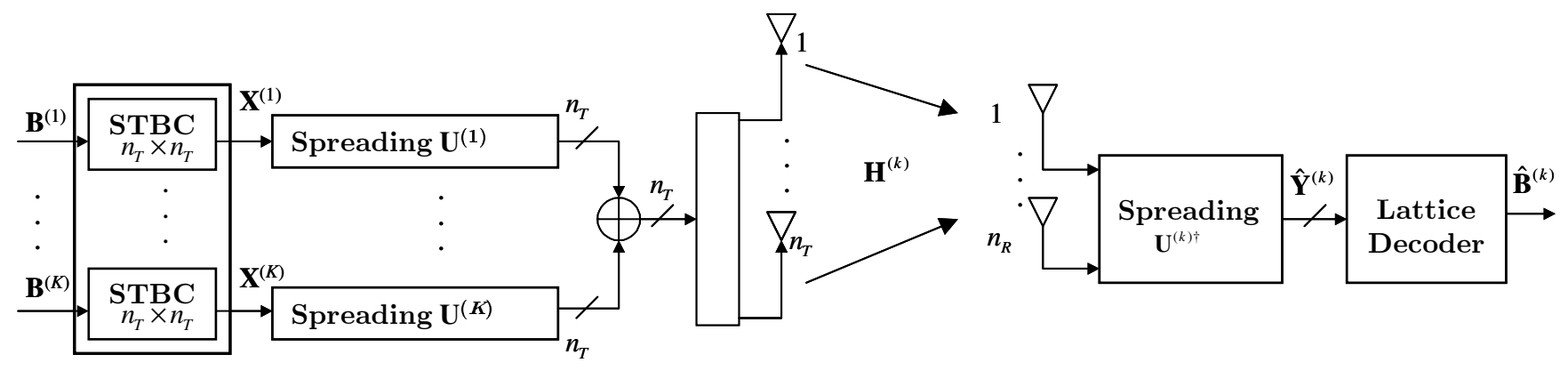

Base Station

$\boldsymbol{k}$-th User Receiver

Fig. 1. A MIMO Multiuser System

Since the encoder and decoder structures for any user are similar, we only consider the general code construction and lattice decoding for an arbitrary $k$-th user. The superscript $k$ is thus omitted for brevity.

For any $k$-th user, the base station transmits the $n_{T} \times n_{R}$ QAM information symbol matrix

$$
\begin{aligned}
\mathbf{B} & =\left[\begin{array}{cccc}
\mathbf{b}_{1} & \mathbf{b}_{2} & \cdots & \mathbf{b}_{n_{R}}
\end{array}\right] \\
& =\left[\begin{array}{cccc}
b_{1,1} & b_{1,2} & \cdots & b_{1, n_{R}} \\
\vdots & \vdots & \ddots & \vdots \\
b_{n_{T}, 1} & b_{n_{T}, 2} & \cdots & b_{n_{T}, n_{R}}
\end{array}\right]
\end{aligned}
$$

where $b_{i, l} \in \mathbb{Z}[j], i=1, \ldots, n_{T}, l=1, \ldots, n_{R}$.

In this paper, we consider square linear dispersion STBCs $[9,10]$ with full rate and full diversity. The QAM information symbols are encoded by such STBCs into an $n_{T} \times n_{T}$ codeword matrix $\mathbf{X}=\left\{x_{i, l}\right\} \in \mathbb{C}, i, l=1, \ldots, n_{T}$. The codeword matrices for each user are separated by orthogonal spreading matrices. Let $\mathbf{U} \in \mathbb{C}^{n_{T} \times K n_{T}}$ be the orthogonal spreading matrix for user $k$ and $\mathbf{U}^{(l)} \in \mathbb{C}^{n_{T} \times K n_{T}}$ be the one for another user $l$, where $l \neq k$. We have

$$
\mathbf{U U}^{\dagger}=\mathbf{I}_{n_{T}} \quad \mathbf{U}^{(l)} \mathbf{U}^{\dagger}=0,
$$

The orthogonal signature matrices can be chosen, for example, as submatrices of a discrete Fourier transform (DFT) matrix or Hadamard matrix.

We assume that perfect CSI is known at the receiver of each user. The $k$-th user receives the signal matrix $\mathbf{Y} \in \mathbb{C}^{n_{R} \times K n_{T}}$, which is given by

$$
\mathbf{Y}=\mathbf{H X} \mathbf{U}+\mathbf{H}\left(\sum_{l=1, l \neq k}^{K} \mathbf{X}^{(l)} \mathbf{U}^{(l)}\right)+\mathbf{Z},
$$

where $\mathbf{X}^{(l)}$ is the codeword matrix for the $l$-th user.

In (2), $\mathbf{Z}$ is the complex i.i.d. Gaussian noise matrix with entries $\mathcal{C} \mathcal{N}\left(0, N_{0}\right)$ and $\mathbf{H} \in \mathbb{C}^{n_{R} \times n_{T}}$ is the independent
Rayleigh fading channel matrix with complex i.i.d. entries $\mathcal{C N}(0,1)$, which is given by

$$
\mathbf{H}=\left[\begin{array}{c}
\mathbf{h}_{1} \\
\mathbf{h}_{2} \\
\vdots \\
\mathbf{h}_{n_{R}}
\end{array}\right]=\left[\begin{array}{cccc}
h_{1,1} & h_{1,2} & \cdots & h_{1, n_{T}} \\
h_{2,1} & h_{2,2} & \cdots & h_{2, n_{T}} \\
\vdots & \vdots & \ddots & \vdots \\
h_{n_{R}, 1} & h_{n_{R}, 2} & \cdots & h_{n_{R}, n_{T}}
\end{array}\right]
$$

We assume that the channel matrix $\mathbf{H}$ is constant during one STBC block and varies from one block to another independently.

\section{STBCs FOR MIMO MULTIUSER SYSTEMS}

In this section, we first recall the rank and determinant criteria for STBC design. We show how to use TAST codes and perfect STBCs for the downlink of MIMO multiuser systems.

\section{A. Rank and Determinant Criteria}

For the $k$-th user, we assume that a codeword $\mathbf{X}$ is transmitted. The maximum-likelihood receiver might decide erroneously in favor of another codeword $\hat{\mathbf{X}}$. Let $r$ denote the rank of the codeword difference matrix $\mathbf{C}=\mathbf{X}-\hat{\mathbf{X}}$, and $\lambda_{i}, i=1, \ldots, r$, be the eigenvalues of the codeword distance matrix $\mathbf{A}=\mathbf{C C}^{\dagger}$.

Then the pairwise error probability (PWEP) is upper bounded by

$$
P(\mathbf{X} \rightarrow \hat{\mathbf{X}}) \leq\left(\prod_{i=1}^{r} \lambda_{i}\right)^{-n_{R}}\left(\frac{E_{s}}{N_{0}}\right)^{-r n_{R}}
$$

where $E_{s}$ is the average energy of QAM information symbols and $\frac{E_{s}}{N_{0}}$ denotes the signal-to-noise ratio (SNR) per transmit antenna.

In (3), we call the minimum value of $r n_{R}$ the diversity gain. We call the minimum value of $\left(\prod_{i=1}^{r} \lambda_{i}\right)^{1 / r}$ the coding gain. In order to minimize the PWEP, the rank and determinant criteria in [1] were proposed as follows: 
- Rank Criterion: The minimum rank $r$ of the codeword difference matrix should be maximized;

- Determinant Criterion: The minimum determinant $\prod_{i=1}^{r} \lambda_{i}$ should be maximized.

In our MIMO multiuser system, we consider the TAST and perfect STBCs that achieve full diversity $n_{R} n_{T}$ and full rate of $n_{T}$ QAM symbols per channel use.

\section{B. TAST Codes for the Downlink of Multiuser MIMO Systems}

We recall TAST codes from [6]. The TAST codes are constructed by transmitting a scaled DAST code in each layer (or thread) $l$, where $l=1, \ldots, n_{R}$, i.e.

$$
\mathbf{x}_{l}=\phi_{l} \mathbf{M b}_{l},
$$

where $\mathbf{x}_{l}$ are the encoded symbols, $\mathbf{b}_{l}$, are the complex QAM symbol vectors defined in (1), and $\phi_{l}$ is chosen to ensure full diversity and maximize the coding gain of the component codes. In [6], $\phi_{l}$ is given by

$$
\phi_{l}=\phi^{(l-1) / n_{T}},
$$

where $\phi=e^{i \lambda}$ and $\lambda \neq 0$ is either an algebraic number or transcendental number [6].

In (4), $\mathbf{M} \in \mathbb{C}^{n_{T} \times n_{T}}$ is a rotation matrix defining a DAST code. Let $\mathbf{s}=\left[s_{1}, \ldots, s_{n_{T}}\right]^{T}=\mathbf{M b}$ and $\hat{\mathbf{s}}=\left[\hat{s}_{1}, \ldots, \hat{s}_{n_{T}}\right]^{T}$ $=$ M $\hat{\mathbf{b}}$ be two different DAST codewords, where $\mathbf{b}$ and $\hat{\mathbf{b}}$ are two different information symbol vectors. The rotation matrix $\mathbf{M}$ is chosen to maximize the associated minimum product distance $d_{p}$, defined as [11],

$$
d_{p}=\min _{\mathbf{s} \neq \mathbf{s}} \prod_{i=1}^{n_{T}}\left|s_{i}-\hat{s}_{i}\right| .
$$

Thus one can easily verify that DAST codes achieve full diversity, and their coding gains are proportional to the minimum product distance associated with the rotations used. The rotation matrix $\mathbf{M}$ is constructed from an algebraic number field $\mathbb{Q}(\theta)$ of degree $n_{T}[6,12]$.

For $L$ layers, where $L=n_{R}$ for the system in this paper, we can write the TAST codeword matrix as

$$
\mathbf{X}=\sum_{l=1}^{n_{R}}\left(\phi_{l} \mathbf{e}^{l-1}\right) \operatorname{diag}\left(\mathbf{M b}_{l}\right),
$$

where

$$
\mathbf{e}=\left[\begin{array}{cccccc}
0 & 1 & 0 & \cdots & 0 & 0 \\
\vdots & 0 & 1 & 0 & \cdots & 0 \\
\vdots & \vdots & \ddots & \ddots & \ddots & \vdots \\
0 & 0 & 0 & 0 & 1 & 0 \\
0 & 0 & 0 & 0 & \cdots & 1 \\
1 & 0 & 0 & 0 & \cdots & 0
\end{array}\right]
$$

C. Perfect STBCs for the Downlink of Multiuser MIMO Systems

For the special cases of $n_{T}=3,4,6$, perfect STBCs was proposed in $[9,10]$. The perfect STBCs are constructed based on cyclic division algebras, where the codeword is given by $[9,10]$,

$$
\mathbf{X}=\sum_{l=1}^{n_{T}} \mathbf{e}^{l-1} \operatorname{diag}\left(\mathbf{M b}_{l}\right),
$$

where

$$
\mathbf{e}=\left[\begin{array}{ccccc}
0 & 1 & 0 & \cdots & 0 \\
\vdots & 0 & 1 & 0 & \vdots \\
\vdots & & & \ddots & \vdots \\
0 & 0 & 0 & 0 & 1 \\
\gamma & 0 & 0 & 0 & 0
\end{array}\right],
$$

and $\gamma$ is chosen from $\mathbb{Z}[j]$ in order to achieve the full diversity and non-vanishing determinant [9]. Comparing to TAST codes, we have a different e matrix and $\phi=1$.

In this case, we only consider subcodes of the perfect STBCs with a reduced number of layers $n_{R}$, i.e.,

$$
\mathbf{X}=\sum_{l=1}^{n_{R}} \mathbf{e}^{l-1} \operatorname{diag}\left(\mathbf{M b}_{l}\right) .
$$

\section{Lattice Decoder for the Downlink of MiMo MULTIUSER SYSTEMS}

The $k$-th user received signal matrix $\mathbf{Y}$ in (2) is despread by multiplying $\mathbf{U}^{\dagger}$, which yields

$$
\hat{\mathbf{Y}}=\mathbf{Y} \mathbf{U}^{\dagger}=\mathbf{H X}+\hat{\mathbf{Z}}
$$

where

$$
\hat{\mathbf{Y}}=\left[\begin{array}{cccc}
\hat{y}_{1,1} & \hat{y}_{1,2} & \cdots & \hat{y}_{1, n_{T}} \\
\vdots & \vdots & \ddots & \vdots \\
\hat{y}_{n_{R}, 1} & \hat{y}_{n_{R}, 2} & \cdots & \hat{y}_{n_{R}, n_{T}}
\end{array}\right]
$$

and

$$
\hat{\mathbf{Z}}=\mathbf{Z} \mathbf{U}^{\dagger}=\left[\begin{array}{cccc}
\hat{z}_{1,1} & \hat{z}_{1,2} & \cdots & \hat{z}_{1, n_{T}} \\
\vdots & \vdots & \ddots & \vdots \\
\hat{z}_{n_{R}, 1} & \hat{z}_{n_{R}, 2} & \cdots & \hat{z}_{n_{R}, n_{T}}
\end{array}\right]
$$

Let us define

$$
\tilde{\mathbf{M}}=\mathbf{M} \otimes \mathbf{I}_{n_{R}} \quad \tilde{\mathbf{X}}=\tilde{\mathbf{M}} \times \operatorname{vec}(\mathbf{B}),
$$

where $\otimes$ denotes matrix Kronecker product and

$$
\operatorname{vec}(\mathbf{B})=\left[\begin{array}{c}
\mathbf{b}_{1} \\
\mathbf{b}_{2} \\
\vdots \\
\mathbf{b}_{n_{R}}
\end{array}\right] .
$$

Vectorizing (11) yields

$$
\operatorname{vec}(\hat{\mathbf{Y}})=\operatorname{vec}(\mathbf{H X})+\operatorname{vec}(\hat{\mathbf{Z}}),
$$

where

$$
\operatorname{vec}(\hat{\mathbf{Y}})=\left[\hat{y}_{1,1}, \ldots, \hat{y}_{1, n_{T}}, \ldots, \hat{y}_{n_{R}, 1}, \ldots, \hat{y}_{n_{R}, n_{T}}\right]^{T},
$$




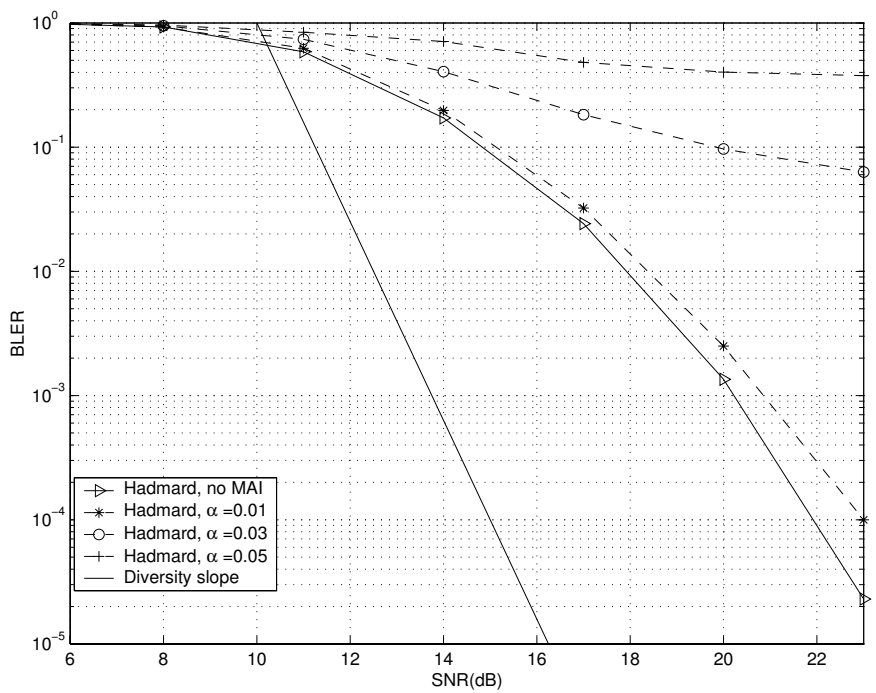

Fig. 2. The Downlink of MIMO Multiuser System 1

$$
\operatorname{vec}(\hat{\mathbf{Z}})=\left[\hat{z}_{1,1}, \ldots, \hat{z}_{1, n_{T}}, \ldots, \hat{z}_{n_{R}, 1}, \ldots, \hat{z}_{n_{R}, n_{T}}\right]^{T}
$$

and $\operatorname{vec}(\mathbf{H X})=\tilde{\mathbf{H}} \tilde{\mathbf{X}}$ is given in (17). For perfect STBC, e is given in (10) and $\phi=1$.

Substituting (14) and (17) into (16) yields

$$
\operatorname{vec}(\hat{\mathbf{Y}})=\tilde{\mathbf{T}} \operatorname{vec}(\mathbf{B})+\operatorname{vec}(\hat{\mathbf{Z}}),
$$

where $\tilde{\mathbf{T}}=\tilde{\mathbf{H}} \tilde{\mathbf{M}}$.

Let us define

$$
\mathcal{Y}=[\Re(\operatorname{vec}(\hat{\mathbf{Y}})) \Im(\operatorname{vec}(\hat{\mathbf{Y}}))]^{T},
$$

where $\Re(\cdot)$ and $\Im(\cdot)$ denote the real and imaginary part of the symbol vectors. Let us define

$$
\mathcal{B}=[\Re(\operatorname{vec}(\mathbf{B})) \Im(\operatorname{vec}(\mathbf{B}))]^{T}
$$

and

$$
\mathcal{H}=\left[\begin{array}{cc}
\Re(\tilde{\mathbf{T}}) & -\Im(\tilde{\mathbf{T}}) \\
\Im(\tilde{\mathbf{T}}) & \Re(\tilde{\mathbf{T}})
\end{array}\right]
$$

Lattice decoding can be employed to decode the $k$-th user QAM symbols $\hat{\mathcal{B}}$, i.e.

$$
\hat{\mathcal{B}}=\underset{\mathbf{B} \in \mathcal{S}^{n}}{\arg \min }\|\hat{\mathcal{Y}}-\mathcal{H} \hat{\mathcal{B}}\|^{2},
$$

where $n=2 \times n_{T} \times n_{R}$.

Remark: The choice of the $n_{R}$ layers within the codeword $\mathrm{X}$ is irrelevant, since this corresponds to a permutation of the blocks in the matrix $\tilde{\mathbf{H}}$ which yields an equivalent code.

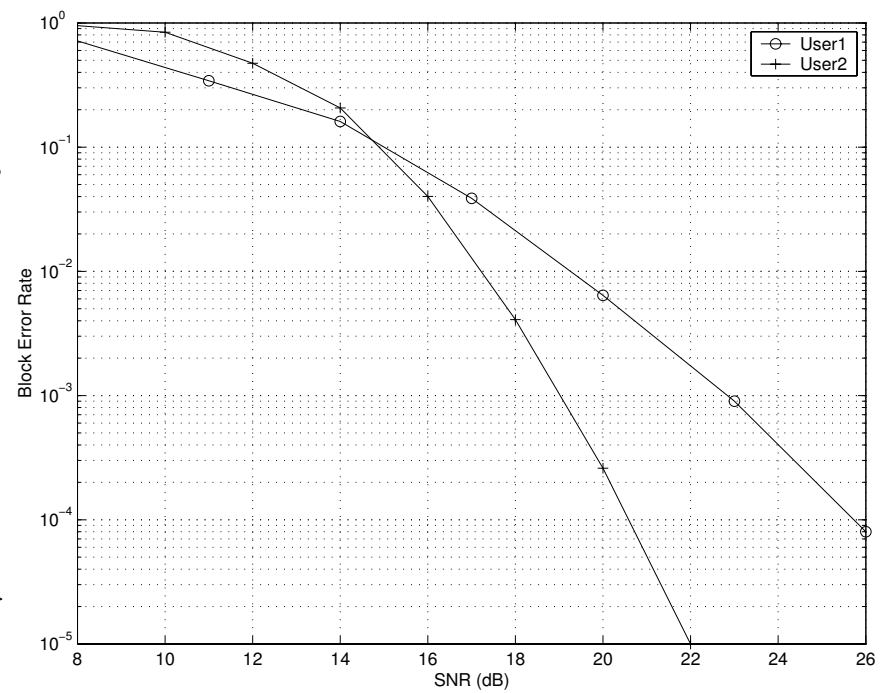

Fig. 3. The Downlink of MIMO Multiuser System 2

\section{Simulation Results}

In the simulations, we show performance of the downlink MIMO system employing perfect STBCs. Performance is evaluated in terms of BLER by Monte Carlo simulation. The $\mathrm{SNR}$ per user is defined as $\mathrm{SNR}=n_{R} E_{s} / N_{0}$. In the simulation, we consider two different systems as below.

System 1: We assume that the base station has four transmit antennas. There are two users, each of which has two receive antennas and 16-QAM symbols are used. We assume each STBC block consists of 8 symbols per user.

We choose $4 \times 4$ perfect STBC using two layers, where the rotation matrix $\mathbf{M}$ is given in $[9,10]$ and $\gamma^{2}=-1$. This provides full diversity and highest possible rate of $n_{R} n_{T}=8$ symbols per block per user. We choose Hadamard matrices as the spreading matrices.

Performance of System 1 is shown in Fig. 2 together with the diversity gain slope. Due to multipath propagation, different time delayed versions of the transmitted signal may result in MAI, which destroys the orthogonality of the spreading codes. In Fig. 2, we show the impact of MAI on the code performance. For User 1, after despreading, we have $\mathbf{U}^{(2)} \mathbf{U}^{(1)}=\alpha \mathbf{1}_{n_{T}}$, where the MAI interference coefficient is chosen to be $\alpha=0.01,0.03,0.05$, respectively. We can see an error floor appears when $\alpha>0$.

System 2: We assume that the base station has four transmit antennas. There are two users: User 1 has one receive antenna, User 2 has three receive antennas, and 16-QAM symbols are used.

The total transmission rate per block is 16 symbols. The transmitted codeword consists of one layer perfect STBC for User 1 (4 QAM symbols), and three layer perfect STBC for User 2 (12 QAM symbols). The performance of System 2 is shown in Fig. 3. We notice that User 2 achieves better 


$$
\operatorname{vec}(\mathbf{H X})=\tilde{\mathbf{H}} \tilde{\mathbf{X}}=\left[\begin{array}{cccc}
\operatorname{diag}\left(\mathbf{h}_{1}\right) & \left(\operatorname{diag}\left(\mathbf{h}_{1}\right) \phi \mathbf{e}\right)^{T} & \cdots & \left(\operatorname{diag}\left(\mathbf{h}_{1}\right) \phi^{n_{R}-1} \mathbf{e}^{n_{R}-1}\right)^{T} \\
\vdots & \vdots & \ddots & \vdots \\
\operatorname{diag}\left(\mathbf{h}_{l}\right) & \left(\operatorname{diag}\left(\mathbf{h}_{l}\right) \phi \mathbf{e}\right)^{T} & \cdots & \left(\operatorname{diag}\left(\mathbf{h}_{l}\right) \phi^{n_{R}-1} \mathbf{e}^{n_{R}-1}\right)^{T} \\
\vdots & \vdots & \ddots & \vdots \\
\operatorname{diag}\left(\mathbf{h}_{n_{R}}\right) & \left(\operatorname{diag}\left(\mathbf{h}_{n_{R}}\right) \phi \mathbf{e}\right)^{T} & & \left(\operatorname{diag}\left(\mathbf{h}_{n_{R}}\right) \phi^{n_{R}-1} \mathbf{e}^{n_{R}-1}\right)^{T}
\end{array}\right]\left[\begin{array}{c}
x_{1,1} \\
x_{1,2} \\
\vdots \\
x_{1, n_{T}} \\
\vdots \\
x_{l, 1} \\
x_{l, 2} \\
\vdots \\
x_{l, n_{T}} \\
\vdots \\
x_{n_{R}, 1} \\
x_{n_{R}, 2} \\
\vdots \\
x_{n_{R}, n_{T}}
\end{array}\right]
$$

performance at higher SNRs thanks to its receive antenna diversity, even if its rate is three times the rate of User 1.

Remarks: To the authors' knowledge, no comparable scheme was found in the literature for comparison. The proposed scheme offers great flexibility in the number of terminal receive antennas.

\section{CONCLUSION}

We consider space time block codes for the downlink of a multiuser MIMO system in this paper. We show how to use TAST block codes and perfect STBCs for such systems in order to achieve the highest possible transmission rate and full diversity gain. Orthogonal spreading matrices are used to separate the data streams of multiple users. At the receiver of each user, maximum likelihood decoding is obtained by using a lattice decoder. Performance of the space-time block coded system with different MAI is evaluated by simulations. It is shown that the system has good performance when the interference coefficient $\alpha=0$, otherwise, an error floor appears. The proposed scheme is suitable to a network supporting different types of terminals due to its flexibility.

\section{ACKNOWLEDGMENT}

This work is supported by the STREP project No. IST026905 (MASCOT) within the sixth framework programme of the European Commission, Australian Research Council (ARC) Project DP 0663567 and ARC Communications Research Work (ACoRN) RN 0459498.

\section{REFERENCES}

[1] V. Tarokh, N. Seshadri and A. R. Calderbank, "Space-Time Codes for High Data Rate Wireless Communications: Performance Criterion and Code Construction," IEEE Transactions on Information Theory, vol. 44, no. 2, pp. 744-765, 1998.

[2] S. M. Alamouti, "A simple transmit diversity technique for wireless communications," IEEE Journals of Selected Areas on Communications, vol. 16, no. 8, pp. 1451-1458, Oct. 1998.
[3] V. Tarokh, H. Jafarkhani and A. R. Calderbank, "Space-time block codes from orthogonal designs," IEEE Transactions on Information Theory, vol. 45, no. 5, pp. 1456-1467, July 1999.

[4] M. O. Damen, K. Abed-Meraim, and J.-C. Belfiore, "Diagonal algebraic space-time block codes," IEEE Transactions on Information Theory, vol. 48, pp. 628-636, Mar. 2002.

[5] M. O. Damen, K. Abed-Meraim, and J.-C. Belfiore, "Transmit diversity using rotated constellations with Hadamard transform," IEEE Proc. 2000 Symp. Adaptive Systems for Signal Processing, Communications, and Control, AB, Canada, pp. 396-401, Oct. 2000.

[6] H. El Gamal and M. O. Damen, "Universal space-time codes," IEEE Transactions on Information Theory, vol. 49, no. 5, pp. 1097-1119, May 2003.

[7] B. A. Sethuraman, B. S. Rajan, and V. Shashidhar, "Full-diversity, highrate space-time block codes from division algebras," IEEE Transactions on Information Theory, vol. 49, pp. 2596-2616, Oct. 2003.

[8] J.-C. Belfiore, G. Rekaya, and E. Viterbo, "The Golden Code: A $2 \times$ 2 full-rate space-time code with non-vanishing determinants," IEEE Transactions on Information Theory, vol. 51, no. 4, pp. 1432-1436, Apr. 2005.

[9] G. Rekaya, J.-C. Belfiore, and E. Viterbo, "Algebraic $3 \times 3,4 \times 4$ and $6 \times 6$ space-time codes with non-vanishing determinants," IEEE proceedings of International Symposium on Information Theory and its applications, Parma, Italy, pp. 325-329, Oct. 2004.

[10] F. Oggier, G. Rekaya, J.-C. Belfiore, and E. Viterbo, "Perfect space time block codes," to appear in IEEE Transactions on Information Theory, April. 2006.

[11] X. Girand, E. Boutillon, and J.-C. Belfiore, "Algebraic tools to build modulation schemes for fading channels," IEEE Transactions on Information Theory, vol. 43, pp. 938-952, May 1997.

[12] J. Boutros and E. Viterbo, "Signal space diversity: a power and bandwidth efficient diversity technique for the Rayleigh fading channel," IEEE Transactions on Information Theory, vol. 44, pp. 1453-1467, July 1998.

[13] F. Oggier and E. Viterbo, "Algebraic number theory and code design for Rayleigh fading channels," Foundations and Trends in Communications and Information Theory, vol. 1, pp. 333-415, 2004.

[14] L. T. Berger and L. Schumacher, "Modified space-time transmission in DS-CDMA downlink facilitating MISO channel equalization," IEEE 56th Vehicular Technology Conference, Vancouver, BC, Canada, vol. 2, pp. 941-945, Sept. 2002. 